\title{
THE DESIGN STUDIO: A CASE STUDY IN TEACHING AND LEARNING PRACTICAL KNOWLEDGE
}

\author{
Pau de Solà-Morales \\ Escola Tècnica Superior d'Arquitectura de Reus \\ Universitat Rovira i Virgili \\ Av. de la Universitat, 1 - 43204 Reus \\ pau.desolamorales@urv.cat
}

\begin{abstract}
RESUMEN
¿Qué es el taller de diseño? ¿Cómo funciona? ¿Cuáles son sus fundamentos psicológicos y cognitivos? ¿Está actualizado y adaptado al s.XXI, a sus delirios tecnológicos y a sus cambios pedagógicos? El taller de diseño se puede entender como un constructo que en parte actualiza y en parte continúa el sistema pedagógico que se creó en la Académie Royale en el siglo XVII, y que ha sobrevivido a través de la École de Beaux-Arts, las Escuelas Politécnicas y la Bauhaus, hasta nuestros días. Este artículo trata de estudiar y reunir la bibliografía existente sobre el design thinking y actualizarla a los conocimientos de nuestros días. Se trata de una primera aproximación para explorar y exponer las esencias del taller de diseño de hoy en día; para ver si se adapta como sistema pedagógico a nuestros métodos académicos modernos basados en competencias y al EEES; y para evaluar su relevancia en la actualidad en el aprendizaje de nuestra (y sobre todo de otras) disciplinas, que están buscando desesperadamente formas de capturar y transmitir sus conocimientos prácticos.
\end{abstract}

Palabras clave: taller de proyectos, diseño, arquitectura, design thinking, educación, docencia.

\begin{abstract}
What is the design studio? How does it function? What are its psychological and cognitive foundations? Is it updated and adapted to the 21st c., its technological frenzies and its pedagogical changes? The design studio can be understood as a construct that in part updates, and in part continues, the pedagogical system that was set up at the Académie Royale in the 18th century, and that has survived through the Beaux Arts, the polytechnic and the Bauhaus schools, to our days. This paper seeks to study and pull together the literature on design thinking and update it to current-day knowledge. It is a first approach to explore and expose the essence of the design studio today; to see if it is adapted as a pedagogical system to our modern academic competence-based apprenticeships, and to the EEES; and to evaluate its relevance today in the learning of our and especially of other disciplines, which are desperately looking for ways to capture and convey their practical knowledge.
\end{abstract}

Keywords: design studio, design, architecture, design thinking, education, teaching. 


\section{INTRODUCCIÓN}

¿Qué es el taller de diseño (design studio)? ¿Cómo funciona? ¿Cuáles son sus fundamentos psicológicos y cognitivos? ¿Está adaptado al siglo XXI, sus desvaríos tecnológicos y sus cambios pedagógicos?

Para responder a estas preguntas es necesario empezar enmarcando el problema. El "diseño" (en su acepción anglosajona, design) incluye a disciplinas diferentes pero que aproximadamente abordan los problemas de una manera similar: la arquitectura, el grafismo, el diseño industrial o el diseño de productos, entre otras). Decía Mario Bunge, el gran filósofo de la ciencia que

"mientras que la Ciencia intenta descubrir qué ocurrirá cuando se den ciertas circunstancias, la Tecnología aspira a modificar precisamente esas circunstancias para que ciertas cosas ocurran. La ciencia es analítica, mientras que la tecnología es sintética." [1]

Sin rasgarnos las vestiduras ni sentirnos menos de lo que éramos, tenemos que aceptar que el diseño entra claramente en la categoría de "tecnología": su cometido es crear estructuras y objetos útiles allí donde sólo había materiales o naturaleza. Crear en el sentido de sintetizar, de generar ex novo, de forjar, formar o elaborar algo nuevo dónde antes no había nada. Por lo tanto el diseño es tecnología: la modificación de las circunstancias ambientales para conseguir que determinados fenómenos ocurran. Además, el diseño es un mecanismo de innovación fundamental, poco reconocido como metodología para fomentar la creación de nuevos productos y procesos. [2]

El conocimiento en profundidad del "método de diseño" es fundamental para poder formalizarlo y así poder enseñarlo y reproducirlo como sistema pedagógico: formar a los nuevos diseñadores, así como a los nuevos innovadores es, en principio, algo deseable. $Y$ sin embargo éste es un método harto desconocido y que se reproduce a si mismo más por el "boca-oreja" y por alguna trasmisión oral y experiencial que a través de su comprensión, formalización y trasmisión como método pedagógico. Nadie, o muy poca gente han abordado el problema de la metodología del diseño y la innovación como metodología docente o académica.

\section{ORÍGENES Y FUNCIONAMIENTO}

El taller de diseño se puede entender como una construcción que actualiza en parte, y en parte continúa, el sistema pedagógico que creó el rey francés Louis XIV (el Rey Sol), quién entendió la necesidad de formar a los arquitectos, artesanos, artistas y constructores que trabajaban para su corte, en el marco una gran operación de unificar en un único estilo nacional todas sus creaciones artísticas. Así nació la Académie Royale d'Architecture en el siglo XVII, que después de la Revolución Francesa fue sustituida por la École Polythecnhique y posteriormente fue reinstituida como École Nationale Supérieure des Beax-Arts. Su sistema pedagógico sobrevivió hasta nuestros días con pocas transformaciones a través de las escuelas de arquitectura modernas, la Bauhaus y las escuelas de diseño.

Sus fundamentos son tan simples y tan antiguos como la artesanía y el conocimiento: la relación "maestro-aprendiz". En ella, el diseñador experto y 
experimentado plantea problemas de diseño calculados y controlados al aprendiz no cualificado, y le permite resolverlos por su cuenta con la debida orientación y observación. El aprendiz, por su parte, propone soluciones y aprende sobre la marcha, y en este proceso de aprendizaje se somete a varios ciclos de crítica y corrección de la mano del maestro (o maestros), aprende cómo se pueden resolver algunas de las situaciones que surgen durante el proceso de diseño, y adopta las actitudes correctas hacia las estrategias de composición.

Por lo tanto, podríamos clasificar inicialmente el conocimiento creado en estas sesiones cómo un tipo de conocimiento entre lo cognitivo y lo procedimental, en lo que podríamos calificar de conocimiento práctico o practical knowledge.

\section{FUNDAMENTOS PSICOPEDAGÓGICOS}

El conocimiento trasmitido y adquirido en el taller de diseño no ha sido siempre conocido. Fue estudiado inicialmente por Simon y Newell en los años 1950's y descrito entre otros en su famoso libro (y que tuvo una importante repercusión) The Sciences of the Artificial, [3] que dieron a conocer el trabajo realizado por los diseñadores como problem solving.

El problem solving supone que hay problemas bien definidos, en términos de variables, rangos de objetivos y restricciones. Desde este punto de partida, existe un espacio de soluciones en el cual algunos de los estados particulares (o puntos en ese espacio) son soluciones al problema planteado, mientras que el resto de puntos caen fuera de los límites impuestos por el problema. Además, siempre según Simon y Allen existe un proceso generativo que crea, busca y evalúa dichas soluciones. El método de diseño queda así reducido a un proceso analítico y deductivo, ejecutado por máquinas o por cerebros asimilables a máquinas. [4]

Sin embargo, otras visiones y otros autores posteriores [5] introdujeron la noción de abducción (primero introducida por J.S. Peirce) para poder distinguir a los procesos de diseño de los otros procesos lógico-deductivos más cercanos al método científico o a los métodos computacionales. En la abducción, en particular en la abducción creativa, los problemas son mal o muy mal definidos ("ill-defined") y la solución al problema de diseño viene acompañada necesariamente por la propia formulación del problema. Es decir, no hay inicialmente un problema definido y claro, hasta que hay una solución en ciernes. El proceso de diseño, entonces, además de proponer un dueto problemasolución en forma de una "hipótesis plausible", comprueba si esa hipótesis es válida como solución y como problema a la vez.

El confuso mecanismo de la abducción creativa fue también estudiado por Roozenburg, [6] quien describió el complejo mecanismo o patrón de razonamiento que lleva a nuevas soluciones para los problemas de diseño.

Finalmente, cabe destacar que muchos autores han explicado la importancia de la experiencia y el contexto en la aplicación del conocimiento práctico. Así, un experto es aquél que no aplica reglas en su quehacer diario, sino que ejercita el método resumido más arriba de una manera espontánea. En cambio, el "novato" o el aprendiz son los que se ven obligados a aplicar reglas simples, reglas aprendidas o impuestas, para poder avanzar en las distintas fases de decisión dentro del diseño de un elemento. 
"...one has to abandon the traditional view that a beginner starts with specific cases and, as he becomes more proficient, abstracts and interiorizes more and more sophisticated rules (...) Skill acquisition moves in just the opposite direction -from abstract rules to particular cases. It seems that a beginner makes inferencing using rules and facts just a heuristically programmed computer, but with talent and a great deal of involved experience the beginner develops into an expert who intuitively sees what to do without applying rules." [7]

Esta visión aleja aún más el proceso de diseño de un proceso lógico-deductivo o computacional, dónde las reglas son parte de la creación de nueva información.

Podríamos introducir en la discusión los estudios de caso, que en el entorno de diseño se han denominado "case-based reasoning", como una secuela o potencial ampliación del método lógico deductivo más allá de los límites del propio sistema, extendiéndolo con una base de conocimiento externa. Los estudios de caso tienen también un buen predicamento como metodologías docentes en determinadas disciplinas, aunque suponen un tipo de aprendizaje más "pasivo" frente al aprendizaje activo que el taller de diseño representa. Pero se demuestra que el problema es el mismo, ya que la construcción de la base de conocimiento necesaria para apoyar al proceso de diseño es excesivamente vasta y lenta, y depende de un proceso de formalización simbólico de difícil concreción.

Últimamente, las ciencias de la complejidad y los estudios neurocientíficos y cognitivos han arrojado algo de luz nueva al problema de la inteligencia, la creatividad y la innovación, desde una visión más conexionista, relacionando el nuevo conocimiento con la interconexión de zonas del cerebro, la experimentación y la construcción del conocimiento (constructivismo). El caso es que como humanos aprendemos asociando información nueva y desconocida a información vieja y conocida, o que construimos información nueva sobre información vieja. El conocimiento, aunque sea práctico, se construye como resultado de la actividad constructiva del estudiante, y a través de su comportamiento activo: el estudiante aprende lo que hace. En cambio, el conocimiento no se construye con la transmisión por parte del profesor de unos conocimientos preestablecidos, ni tampoco se construyen mediante la deducción lógica a partir de los conocimientos preexistentes. [8]

En cualquier caso, esta metodología, que hoy se enmarcan dentro de las enseñanzas de "learn-by-doing" o que es próxima a las experiencias de "projectbased learning", que pone a la experiencia como base del aprendizaje, el conocimiento y la inteligencia. Se trata, sin duda, de una metodología que conocen bien todos los arquitectos y diseñadores, aunque sólo de manera empírica. Sin embargo, el método de diseño, el método pedagógico que entra en juego en el taller de diseño no es ninguna de las metodologías docentes al uso, ni tampoco existe una descripción correcta y en profundidad de su funcionamiento. 


\section{OBJETIVAR EL TALLER DE DISEÑO}

¿Cuál es el problema entonces, al que aspiramos contribuir mediante esta actualización de los estudios sobre el design thinking y el taller de diseño? El de poder dar un soporte y una formación a los educadores y profesores de las escuelas de arquitectura, diseño y otras disciplinas. También deseamos poder comprender (que no modificar) cómo funciona el taller de diseño para poderlo exportar a otras disciplinas como metodología docente. Si bien en la arquitectura y el diseño el taller es algo habitual, es probable que muchas otras disciplinas deseen implementar métodos de trabajo y de aprendizaje de los procesos de diseño e innovación que a nosotros nos son naturales.

Se trataría pues, de encontrar la manera de "objetivar" el taller de proyecto como metodología docente, en base a los parámetros expresados más arriba y de actualizarlo a los métodos docentes y de formación y evaluación por competencias. Es necesario entender que esta objetivación debe empezar por hacer un listado exhaustivo de los objetivos docentes del taller, y de las competencias que en él se pueden trabajar. Desde esta primera lista de objetivos, puede trazarse el conjunto de resultados de aprendizaje que el taller puede desarrollar como material de evaluación. Estas dos o tres estructuras serán la base para una correcta programación del taller, mediante la asignación cruzada de objetivos, contenidos, competencias y resultados etc., siendo la metodología la parte invariante del proceso. Sin embargo, el desconocimiento de tal metodología, imposibilitará hacer correctamente la programación de los talleres.

Finalmente, conocer el contenido del taller de proyectos y su funcionamiento interno (es decir, objetivar el taller de diseño) nos permitirá solventar uno de los problemas más acuciantes al que está sometido: la evaluación de los proyectos de los estudiantes. En efecto, comprender bien su funcionamiento debería bastar para realizar una buena programación docente, pero además ambas cosas permiten también que cada uno de los objetivos y/o resultados de aprendizaje sean itemizados y evaluados por separado. La trascripción de estos objetivos y sus niveles en una rúbrica (o sistema evaluativo similar) permite objetivizar también el juicio sobre el trabajo realizado por los estudiantes, de manera que la evaluación global de un proyecto no sea más percibida como una acción arbitraria y sin sentido, sino como el resultado lógico de un proceso evaluativo que da retroacción al estudiante y retroalimenta al sistema educativo.

\section{Notas:}

[1] (Bunge 1967)

[2] Véase por ejemplo mi trabajo presentado en (Solà-Morales 2009)

[3] Véase (Simon 1981)

[4] Para una explicación más rica y en profundidad, referimos al lector a (Rowe 1987)

[5] Véase la definición de abducción dada por (Roozenburg 1992)

[6] (Roozenburg 1993)

[7] (Dreyfus, Dreyfus et al. 1986)

[8] Ralph W. Tyler. (1949) Basic principles of curriculum and instruction. Chicago: The University of Chicago Press. Citado en Teaching Teaching \& Understanding Understanding (part 2/3). http://www.youtube.com/watch?v=2DMnYxc3ank 


\section{BIBLIOGRAFIA}

Bunge, M. (1967). La Investigación científica. Barcelona, Ariel.

Dreyfus, H. L., S. E. Dreyfus, et al. (1986). Mind over machine : the power of human intuition and expertise in the era of the computer. Oxford, UK, B. Blackwell.

Roozenburg, N. (1992). On the Logic of Innovative Design. Research in design thinking: proceedings of a workshop meeting held at the Faculty of Industrial Design Engineering, Delft University oof Technology, the Netherlands, May 29-31, 1991. N. Cross, K. Dorst and N. Roozenburg. Delft, Delft University Press: 127138.

Roozenburg, N. (1993). "On the pattern of reasoning in innovative design." Design Studies 14(1): 4-18.

Rowe, P. G. (1987). Design thinking. Cambridge, Mass., MIT Press.

Simon, H. A. (1981). The sciences of the artificial. Cambridge, Mass., MIT Press.

Solà-Morales, P. (2009). Investigación en Arquitectura: hacia una definición del marco conceptual. III Jornadas sobre Investigación en Arquitectura y Urbanismo. Madrid. 